\title{
INTERSTELLAR GRAINS IN MUSEUMS?
}

\author{
A. G. W. CAMERON \\ Belfer Graduate School of Science, Yeshiva University, New York, N.Y., U.S.A. \\ and
}

Goddard Institute for Space Studies, NASA, New York, N.Y., U.S.A.

\begin{abstract}
It is argued that carbonaceous chondrites, particularly of type I, are probably collections of interstellar grains which have been mildly transformed through exposure to higher than normal temperatures, resulting in a loss of volatile materials.
\end{abstract}

During this conference there have been many discussions of methods for determining the properties of interstellar grains by means of electromagnetic observations and by investigation of the properties of possible laboratory prototypes. It has not been realized that it may be possible to study interstellar grains directly within the laboratory.

Theoretical investigations of the star formation process indicate that the collapse of an interstellar cloud is likely to take place under very low temperature conditions. The calculated temperature in the interior of a collapsing gas cloud is about $10 \mathrm{~K}$ (Larson, 1969). The temperature in the gas starts to rise only when the gas density becomes high enough so that the gas becomes opaque to the transmission of its own radiation, and the compression goes over from an isothermal stage to an adiabatic stage. M. R. Pine and I have recently constructed models of the primitive solar nebula which may have resulted from such an interstellar collapse process (Cameron and Pine, 1973). I believe that these models are sufficiently general in indicating that the temperature will not rise high enough to evaporate completely the interstellar grains, contained within the gas, beyond about one or two astronomical units from the central spin axis of the primitive solar nebula (Cameron, 1973). The important aspect of this conclusion is that small bodies formed at very much larger distances from the central spin axis are likely to be composed of collections of interstellar grains whose properties have been transformed by varying degrees. Naturally, if we are interested in the laboratory examination of interstellar grains, we must seek material which has suffered the least rise in temperature during the adiabatic compression of the interstellar gases.

Ideally, it would be nice to be able to examine a piece of a comet, which has probably formed at very great distances from the central spin axis of the primitive solar nebula, and in which the temperature has always stayed very low. Unfortunately, it is unlikely that we will obtain any sample of cometary material for some time to come, and therefore we must seek the next most primitive material.

This material is presumably carbonaceous chondritic material. Studies of elemental abundances in such meteorites, as well as of the ratios of the oxygen isotopes, tend to show that carbonaceous chondrites have been accumulated in the primitive solar nebula at a temperature in the vicinity of $350 \mathrm{~K}$. Large amounts of water and carbon 
compounds have been retained, although it is obvious that most of the condensed ice has disappeared, along with ammonia and methane, with only the more complex carbon compounds and water of crystallization being retained at this temperature. The more refractory materials, silicates and metallic oxides, have probably been retained with very little change of properties.

One of the most common substances contained in carbonaceous chondrites is magnetite. Huffman and Stapp (1973) has already pointed out to us that magnetite has some interesting polarization properties which suggest interstellar grain material. It is also interesting that the small magnetite grains tend to have remanent magnetism. Even if this were not the case, the magnetite grains would be interesting as a possible subset of the interstellar grains because the larger ones would be ferromagnetic, and the smaller ones would be superparamagnetic. Thus those interstellar grains which have magnetite cores may play a large role in the polarization of interstellar starlight.

It is likely that interstellar grains are nucleated in cool stellar atmospheres, being condensed and expelled by radiation pressure, or possibly precipitated in rapidly expanding stellar atmospheres such as in nova explosions. Studies of the remanent magnetism of magnetite grains in carbonaceous chondrites tend to indicate that the magnetism was acquired in the presence of a field having a strength of 0.1 to $1 \mathrm{G}$ (Brecher, 1972). Such fields naturally exist in the vicinities of stellar surfaces, but the precipitating substance is likely to be metallic iron rather than magnetite. However, I have been informed by Aviva Brecher, who has carried out a number of these studies, that in her opinion the remanent magnetism would be retained if a magnetized iron grain is oxidized to magnetite.

Thus it appears to me to be natural that the nucleation of some of the interstellar grains may provide magnetic particles, upon which other materials can condense in interstellar space. These particles should be alignable by relatively weak interstellar magnetic fields, and they will be part of any collapsing interstellar cloud which forms stars and planetary systems. Those grains which remain rather far out in any primitive gaseous nebula which is formed, will assemble into material resembling carbonaceous chondrites, with only a loss of the more volatile materials, and without a loss of magnetic properties. Of course, I refer here only to the matrix material in types II or III carbonaceous chondrites, and not to chondrules or inclusions. All of the material in type I carbonaceous chondrites should qualify.

Thus it is possible that meteorite collections in our museums may be very valuable for research on interstellar grains. Thin sections of the matrix material in carbonaceous chondrites cannot be expected to reproduce precisely the optical properties of interstellar grains, because there has been some loss of material by heating, and possibly some mineral changes in the remaining material, but some of the properties should undoubtedly resemble those of interstellar grains if this hypothesis is correct, and if so, then the materials responsible for these properties could be identified with somewhat greater certainty than is done at the present time. I therefore recommend that investigations of the properties of carbonaceous chondrites be carried out with this hypothesis in mind. 


\section{Acknowledgements}

This research has been supported in part by grants from the National Science Foundation and the National Aeronautics and Space Administration.

\section{References}

Brecher, A.: 1972, 'On the Primordial Condensation and Accretion Environment and the Remanent Magnetism of Meteorites, in Proc. IAU Symposium on the Evolutionary and Physical Properties of Meteoroids, in press.

Cameron, A. G. W.: 1973, Icarus 18, 407-450.

Cameron, A. G. W. and Pine, M. R.: 1973, Icarus 18, 377-406.

Huffman, D. R. and Stapp, J. L.: 1973, this volume, p. 297.

Larson, R. B.: 1969, Monthly Notices Roy. Astron. Soc. 145, 271-295. 